\title{
Trans-oral robotic surgery versus coblation tongue base reduction for obstructive sleep apnea syndrome
}

\author{
Wei-Che Lan ${ }^{1}$, Wen-Dien Chang ${ }^{2}$, Ming-Hsui Tsai ${ }^{1,3,4}$, Yung-An Tsou ${ }^{\text {Corresp. } 1,3,4}$ \\ ${ }^{1}$ Department of Otolaryngology Head and Neck Surgery, China Medical University Hospital, Taichung, Taiwan \\ 2 Department of Sport Performance, National Taiwan University of Sport, Taichung, Taiwan \\ 3 School of Medicine, China Medical University, Taichung, Taiwan \\ 4 Department of Audiology and Speech-Language Pathology, Asia University, Taichung, Taiwan \\ Corresponding Author: Yung-An Tsou \\ Email address: D6638@mail.cmuh.org.tw
}

Objectives. To compare the efficacy of trans-oral robotic surgery (TORS) with that of coblation assisted tongue base reduction surgery in patients with obstructive sleep apnea syndrome (OSAS). Subjects and Methods. The medical charts were retrospectively reviewed for all OSAS patients admitted to one institution for surgical intervention between 2012 and 2017. We analyzed 33 cases; 16 patients received TORS and 17 received coblation surgery for tongue base reduction. Both groups received concomitant uvulopalatoplasty. Surgical outcomes were evaluated by comparing the initial polysomnography (PSG) parameters with the follow-up PSG data (at least 3 months after the surgery). Epworth sleepiness scale (ESS) and complications were also compared between the 2 groups. Results. The success rate ( $\geqq 50 \%$ reduction of pre-operative $A H I$ and post-operative $\mathrm{AHI}<20$ ) in the TORS group and coblation group were $50 \%$ and $58 \%$, respectively, and there was no significant difference $(p=.611)$. The AHI (mean \pm SD) reduction in the TORS and coblation groups were $24.9 \pm 26.5$ events/h and $19.4 \pm 24.8$ events/h, respectively; the between-group difference was not significant $(p=.631)$. ESS improvement did not differ significantly between the TORS and coblation groups (3.8 \pm 6.6 and $3.1 \pm 9.2$, respectively, $p=.873)$. The rates of minor complication were higher in the TORS group (50\%) than that of the coblation group (35.3\%) without statistical significance $(p=.393)$. Conclusion. TORS achieved comparable surgical outcomes compared to coblation assisted tongue base reduction surgery in OSAS patients. Multilevel surgery using either TORS or coblation tongue base reduction combi ned with uvulopalatoplasty is an effective approach for the managementof OSAS. 
1 Trans-oral Robotic Surgery versus Coblation Tongue Base Reduction for

2 Obstructive Sleep Apnea Syndrome

3

4 Wei-Che Lan ${ }^{1}$, Wen-Dien Chang' ${ }^{2}$, Ming-Hsui Tsai ${ }^{1}, 3,4$, Yung-An Tsou'1, 3, 4

$5 \quad{ }^{1}$ Department of Otolaryngology Head and Neck Surgery, China Medical University

6 Hospital, Taichung, Taiwan.

7 2Department of Sport Performance, National Taiwan University of Sport, Taichung,

8 Taiwan.

9 3School of Medicine, China Medical University, Taichung, Taiwan.

10 4Department of Audiology and Speech-Language Pathology, Asia University, Taichung,

11 Taiwan.

12

13 Corresponding Author:

14 Yung-An Tsou ${ }^{1,3,4}$

15 Department of Otolaryngology Head and Neck Surgery, China Medical University

16 Hospital, No.2, Yude Rd., North Dist., Taichung City 404, Taiwan.

17 Email: D6638@mail.cmuh.org.tw 


\section{Abstract}

20

21 Objectives. To compare the efficacy of trans-oral robotic surgery (TORS) with that of

22 coblation assisted tongue base reduction surgery in patients with obstructive sleep

23 apnea syndrome (OSAS).

24 Subjects and Methods. The medical charts were retrospectively reviewed for all OSAS

25 patients admitted to one institution for surgical intervention between 2012 and 2017. We analyzed 33 cases; 16 patients received TORS and 17 received coblation surgery for

27 tongue base reduction. Both groups received concomitant uvulopalatoplasty. Surgical outcomes were evaluated by comparing the initial polysomnography (PSG) parameters with the follow-up PSG data (at least 3 months after the surgery). Epworth sleepiness scale (ESS) and complications were also compared between the 2 groups.

Results. The success rate $(\geqq 50 \%$ reduction of pre-operative $\mathrm{AHI}$ and post-operative $\mathrm{AHI}<20$ ) in the TORS group and coblation group were $50 \%$ and $58 \%$, respectively, and

33 there was no significant difference $(p=.611)$. The AHI (mean $\pm S D)$ reduction in the

34 TORS and coblation groups were $24.9 \pm 26.5$ events/h and $19.4 \pm 24.8$ events/h,

35 respectively; the between-group difference was not significant $(p=.631)$. ESS improvement did not differ significantly between the TORS and coblation groups $(3.8 \pm 6.6$ 
37 and $3.1 \pm 9.2$, respectively, $p=.873)$. The rates of minor complication were higher in the

38 TORS group (50\%) than that of the coblation group (35.3\%) without statistical

39 significance $(p=.393)$.

40 Conclusion. TORS achieved comparable surgical outcomes compared to coblation

41 assisted tongue base reduction surgery in OSAS patients. Multilevel surgery using either

42 TORS or coblation tongue base reduction combined with uvulopalatoplasty is an

43 effective approach for the management of OSAS.

45 Introduction

46 Obstructive sleep apnea syndrome (OSAS) is a common disorder which affects 3-7\% of

47 adult men and $2-5 \%$ of adult women.(Punjabi 2008) OSAS results from upper airway

48 collapse during sleep. Clinical symptoms include fragmented sleep and excessive

49 daytime sleepiness.(Semelka, Wilson et al. 2016) Continuous positive airway pressure

50 (CPAP) is thought to be the gold standard treatment for OSAS,(Lucia Spicuzza 2015) but

51 some patients cannot tolerate it and may seek surgical treatment instead.(Kotecha and

52 Hall 2014) Different levels and degrees of obstruction in OSAS patients lead to variable

53 response to surgical intervention.(Koutsourelakis, Safiruddin et al. 2012) In one study,

54 Vroego et al. analyzed the upper airway collapse patterns in patients with sleep- 
55 disordered breathing by using drug-induced sleep endoscopy (DISE) and multilevel

56 collapse was disclosed in $68.2 \%$ of all patients.(Vroegop, Vanderveken et al. 2014) As

57 the intricacies of airway collapse are better understood, due to improvements in

58 diagnostic and evaluative methods, multilevel surgery is becoming a more common

59 method of successfully treating OSAS.(Thaler, Rassekh et al. 2016, Lin, Friedman et al.

60 2017) Among these patients with multilevel collapse, the most frequently seen pattern

61 was the concomitant collapse of palatal and tongue base (25.5\%).(Vroegop,

62 Vanderveken et al. 2014) Uvulopalatopharyngoplasty (UPPP) is the most commonly

63 reported surgery to address oropharyngeal obstruction. For dealing with tongue base

64 obstruction, trans-oral robotic surgery (TORS) and coblation assisted tongue base

65 reduction surgery were two of the most published tongue base tissue reduction

66 procedures.

67

68 Several preoperative assessment strategies have been used. The Friedman tonsil

69 grading scale classifies the tonsil size into five grades (grade 0 -IV) according to the

70 location the tonsil relative to the surrounding structures.(Friedman, Tanyeri et al. 1999)

71 The Friedman tongue position (FTP) grading system is evaluated similarly to the

72 modified Mallampati classification, but the tongue is evaluated in a neutral position 
73 without protrusion. The Friedman staging system incorporates FTP, Friedman tonsil

74 grading scale and BMI to classify OSAS patients into four stages: stage I includes

75 patients with tonsils graded III-IV, FTP graded I-II and BMI<40 kg/M²; stage III includes

76 patients with tonsils graded 0 -II, FTP graded III-IV and BMI<40 kg/M²; stage IV includes

77 patients with $\mathrm{BMI}>40 \mathrm{~kg} / \mathrm{M}^{2}$ or significant craniofacial or other anatomic abnormalities;

78 stage II includes patients beyond stage I, III, IV.(Friedman, Salapatas et al. 2017)

79 Fiberoptic nasopharyngoscopy with Muller's maneuver, which mimics the

80 pathophysiological status of OSAS during wakefulness by asking the patient to block

81 bilateral nostrils and inhale with mouth closed, can identify the level and degree of upper

82 airway collapse.(Terris, Hanasono et al. 2000) Drug-induced sleep endoscopy (DISE),

83 which is recognized as a breakthrough in evaluation of OSAS patients, can provide direct

84 identification of airway collapse during intravenous anesthesia. The VOTE classification

85 is utilized for the findings of DISE.(Kezirian, Hohenhorst et al. 2011)

87 For most patients with oropharyngeal obstruction, uvulopalatopharyngoplasty (UPPP) is

88 one of the most common and effective surgical procedures.(Khan, Ramar et al. 2009)

89 However, oropharyngeal obstruction combined with tongue base obstruction is

90 recognized as an important reason for failure after pharyngoplasty procedures.(Choi, 
91 Cho et al. 2016) For tongue base obstruction, multiple procedures have been proposed

92 and could be simply categorized into tongue base volume reduction and tongue

93 suspension. Among these procedures, trans-oral robotic surgery (TORS) and coblation

94 assisted tongue base reduction surgery proved to be the most published therapeutic

95 methods in the field of tongue base reduction.(Cammaroto, Montevecchi et al. 2017)

96 TORS can provide a 3D visual field and the operator can easily access the tongue base

97 area and perform surgery using delicately controlled robotic instruments. Nevertheless,

98 the high cost of TORS makes operators and patients hesitant touse it.(Cammaroto,

99 Montevecchi et al. 2017) Endoscopic coblation assisted tongue base reduction surgery

100 has been reported to be a useful procedure for tongue base obstruction and it has a

101 lower cost compared to TORS.(Friedman, Hamilton et al. 2012, Li, Lee et al. 2016)

102 However, there is a lack of fair comparison studies regarding the treatment efficacy and

103 safety between TORS and coblation assisted tongue base reduction. Therefore, this

104 study was conducted to compare the subjective and objective outcomes of TORS with

105 endoscope-guided coblation tongue base reduction.

107 Materials and methods

108 Medical charts were retrospectively reviewed for OSAS patients admitted for TORS or 
109 coblation tongue base reduction surgery to a single tertiary hospital between 2012 and

1102017.33 patients with age ranging from 18 to 62 years met the inclusion criteria (Table

111 1). Patients who were excluded were those without available postoperative

112 polysomnography (PSG) data. PSG was performed at 3-12 months after the surgery.

113 Patients who had previous upper airway surgery for OSAS were also excluded. This

114 study was approved by the Institutional Review Board of the China Medical University

115 Hospital (project approval number CMUH103-REC1-078).

116

117 Detailed profiles were constructed for each patient and included the following variables:

118 age, sex, body mass index (BMI), tonsil grade, Friedman tongue position, Friedman

119 stage, pre-operative and post-operative Epworth sleepiness scale (ESS). Post-operative

120 ESS was recorded at the date for post-operative PSG. Nasopharyngoscopy with Muller's

121 maneuver and drug-induced sleep endoscopy (DISE) were performed in all patients to

122 evaluate the site of obstruction and the pattern of the airway collapse. The grades of

123 airway collapse in Muller's maneuver were divided into four grades according to the

124 percentage change in cross-sectional area: grade I $\leqq 25 \%$ collapse, grade II $>25 \%$ and

$125 \leqq 50 \%$ collapse, grade III $>50 \%$ and $\leqq 75 \%$ collapse, grade IV $>75 \%$ collapse. VOTE

126 classification was utilized for reporting DISE findings and the grade of collapse were 
127 classified as 0 ( $<50 \%$ obstruction); 1 ( $50-75 \%$ obstruction) and 2 ( $>75 \%$ obstruction).

128 Patients undergoing surgery must have at least partial tongue base collapse confirmed

129 by Muller's maneuver and DISE. Details were also recorded from pre-operative and

130 post-operative PSG data, and included $\mathrm{AHI}$, apnea index (Al), lowest oxygen saturation

131 (min-SpO2), and cumulative time percentage with SpO2 <90\% (CT90) (Table 2). The

132 success of the surgery was defined as achievement of $\geqq 50 \%$ reduction of pre-operative

$133 \mathrm{AHI}$ and a post-operative $\mathrm{AHI}<20$. Perioperative parameters, including the length of stay

134 in hospital, the numeric rating scale (NRS) assessment on the first postoperative day for

135 pain intensity and complications, were recorded.

136

137 In this study, 16 patients received TORS and 17 patients received coblation surgery for

138 tongue base reduction. All patients received conventional uvulopalatopharyngoplasty

139 combined with tongue base reduction for multilevel obstruction in these patients.

140 All of the surgeries were performed by a single surgeon.

142 The surgical procedure of trans-oral robotic surgery for tongue base volume reduction

143 was performed similar to the previous published literature.(Vicini, Dallan et al. 2010,

144 Friedman, Hamilton et al. 2012) General anesthesia was introduced via nasotracheal 
145 intubation. The anesthesia machine was positioned at the left side foot of the bed. The

146 surgical cart of the da Vinci surgical system (Intuitive Surgical, Sunnyvale, California,

147 USA) approached the patient from the right-hand side with an angle of 45 degrees to the

148 bed. The scrub nurse stood next to the patient's left hand and the first assistant sat at the

149 head of the bed. The operative surgeon was at the operative console and used open-

150 surgery hand movements which were precisely replicated in the operative field by the

151 robotic instruments. The laryngeal advanced retractor system (Fentex, Tuttlingen,

152 Germany) was used to expose the tongue base area. The size of the tongue blade was

153 chosen accordingly to well expose the tongue base. Under 30 degree 3D camera

154 endoscope, tongue base tissue was grasped by robotic forceps and cauterized with

155 spatula monopolar electrode. The midline posterior glossectomy began from the

156 foramen cecum and advanced posteriorly to vallecula without injury to epiglottis mucosa,

157 laterally to $1 \mathrm{~cm}$ from the midline and $1.5 \mathrm{~cm}$ inferior to the tongue base surface.

158

159 Endoscopic coblation assisted tongue base reduction surgery was performed similar to

160 previous reports.(Wee, Tan et al. 2015, Li, Lee et al. 2016) Under general anesthesia

161 with nasotracheal intubation, the Molt mouth gag (Sklar, West Chester, Pennsylvania,

162 USA) was applied to the left side of labial commissure. We placed a silk suture through 
163 the anterior tongue and the silk was held by a Kelly forceps. The first assistant could

164 easily retract the tongue forward by holding the Kelly forceps. A 70 degree rigid

165 endoscope (Karl Storz, Tuttlingen, Germany) was applied to expose the tongue base

166 area and kept in position by an endoscope holder (Karl Storz, Tuttlingen, Germany).

167 With the aid of the endoscope holder, the surgeon could perform the procedure

168 bimanually and thus decrease the operation time and the morbidity related to blood loss.

169 The Coblator II ENT Surgery System and PROCISE MAX coblation wand (Arthrocare

170 ENT, Sunnyvale, California, USA) were used for the midline posterior glossectomy. The

171 targeted resection area was the same as the TORS mentioned above.

172

173 We used the Statistical Packages for the Social Sciences version 24.0 (IBM Corp.;

174 Armonk, NY, USA) for statistical analysis of the data. The descriptive statistic was used

175 to present the outcome values. The Mann-Whitney and signed Wilcoxon test were used

176 for comparing numerical variables between and within groups, respectively. Fisher's

177 exact test was used for categorical variables. A p value of less than .05 was considered

178 to be statistically significant.

179

180 Result 
181 Among the 33 patients in this analysis, 16 were of the TORS group (age of $39.4 \pm 12.3$

182 years; mean \pm SD) and 17 patients were of the coblation group (age of $38.7 \pm 11.5$ years,

183 mean \pm SD). Male comprised $93.8 \%$ in the TORS group and $76.5 \%$ in the coblation

184 group. The mean body mass index (BMI) at the time of admission was $28.2 \pm 3.8 \mathrm{~kg} / \mathrm{m}^{2}$ in

185 the TORS group and $27.4 \pm 5.6 \mathrm{~kg} / \mathrm{m}^{2}$ in the coblation group. There were no significant

186 differences in tonsil grading scale, Friedman tongue position and Friedman staging

187 system between the two groups before surgery. The grades of collapse in Muller's

188 maneuver and DISE were similar in both groups. The mean Epworth sleepiness scale

189 (ESS) was $11.1 \pm 4.7$ in the TORS group and $10.9 \pm 5.2$ in the coblation group. All patients

190 received polysomnography (PSG) for pre-operative evaluation. The mean baseline

191 apnea-hyponea index (AHI) was 50.5 \pm 19.6 events/h and mean apnea index ( $\mathrm{Al}$ ) was

$19233.3 \pm 19.2$ events/h in the TORS group; corresponding values were $44.9 \pm 28.8$ events/h

193 and $31.3 \pm 26.8$ events/h, respectively, in the coblation group. The mean lowest oxygen

194 saturation (min-SpO2) was $73.8 \pm 6.8 \%$ and mean cumulative time percentage with

$195 \mathrm{SpO} 2<90 \%$ (CT90) was $15.1 \pm 14.5 \%$ in the TORS group; corresponding values were

$19674.0 \pm 10.0 \%$ and $13.1 \pm 15.3 \%$ in the coblation group. Demographics and baseline PSG

197 data for both groups are summarized in Table 2. There were no significant between-

198 group differences prior to treatment. 
200 The comparisons within-group (Table 3) and between-group (Table 4) were analyzed,

201 respectively.Statistically significant improvement of ESS was observed in both groups.

202 ESS (mean \pm SD) improvement did not differ significantly between the TORS and

203 coblation groups $(3.4 \pm 3.1$ and $2.8 \pm 4.4$, respectively, $p=.646 ; 95 \% \mathrm{Cl}=-3.30-2.08$,

204 Figure 2). The AHI (mean \pm SD) reduced significantly from $50.5 \pm 19.6$ events/h to

$20525.5 \pm 19.5$ events/h in the TORS group $(p=.002)$. In the coblation group, the mean AHI

206 reduced significantly from $44.8 \pm 28.8$ events/h to $25.5 \pm 23.3$ events/h $(p=.005)$. The AHI

207 reduction in the TORS and coblation groups were significantly reduced, and the

208 between-group difference was not significant $(p=.631 ; 95 \% \mathrm{Cl}=-12.67-23.73$, Figure 3

209 and 4). The mean AI reduced significantly in both TORS and coblation group $(p=.014$ and

$210 p=.004$, respectively), but the mean Al reduction did not differ significantly between the

211 groups as well $(p=.657 ; 95 \% \mathrm{Cl}=-18.53-14.60$, Figure 4$)$. The mean min-SpO2

212 improved from $73.8 \pm 6.8 \%$ to $83.8 \pm 5.6 \%(p=.001)$ in the TORS group and from

$21374.0 \pm 10.0 \%$ to $80.7 \pm 12.6 \%$ in the coblation group $(p=.045)$. The mean improvement of

214 min-SpO2 was $10.0 \pm 7.7 \%$ in the TORS group and $6.7 \pm 12.6 \%$ in the coblation group.

215 There were no statistically significant differences in the improvement of min-SpO2

216 between the two groups $(p=.363 ; 95 \% \mathrm{Cl}=-4.11-10.82$, Figure 5$)$. The TORS group 
217 patients had a greater reduction in CT90 percentage, but the difference was not

218 significant. The success rate in the TORS group and coblation group were $50 \%$ and $58 \%$,

219 respectively, and there was no statistically significant difference $(p=.611 ; 95 \% \mathrm{Cl}=-4.93-$

220 13.79, Figure 5).

221

222 The mean numeric rating scales (NRS) for pain evaluation on the first postoperative day

223 were similar in both groups (TORS group $=2.8 \pm 0.9 ;$ coblation group $=2.5 \pm 0.7 ; p=.533$ ).

224 In the TORS group, the length of stay in hospital was longer compared with the coblation

225 group $(p=.002)$. There were no major complications (e.g., intra-operative or post-

226 operative bleeding, airway compromise, prolonged intubation, pneumonia and

227 pharyngeal laceration, tongue limitation) in either group. No tracheotomies were

228 performed for airway management perioperatively. The rates of minor complication,

229 including transient dysphagia, pharyngeal edema and dysgeusia, in the TORS and

230 coblation groups were 8/16 (50\%) and 6/17(35.3\%), respectively.

231

232 Discussion

233 Our results presented that the surgical outcomes of trans-oral robotic surgery (TORS)

234 were comparable to coblation assisted tongue base reduction surgery in obstructive 
235 sleep apnea syndrome (OSAS) patients. The PSG outcomes and success rate were

236 similar for the TORS and coblation groups.

237

238 Multilevel surgery is thought to be more effective than UPPP for management of patients

239 suffering from OSAS. Because of the better understanding of the complexity of the upper

240 airway collapse during sleep in OSAS patients, surgeons can determine correct surgical

241 management according to the site of obstruction and the pattern of the airway

242 collapse.(Toh, Han et al. 2014, Li, Lee et al. 2016, Thaler, Rassekh et al. 2016)

243

244 In a retrospective study, 25 moderate-to-severe OSAS patients with retropalatal and

245 tongue base obstruction received coblation endoscopic lingual lightening and modified

246 uvulopalatopharyngoplasty (relocation pharyngoplasty). AHI (mean \pm SD) decreased

247 significantly from $45.7 \pm 21.7$ to $12.8 \pm 8.2$ events/hour $(p<.001)$ postoperatively and the

248 overall surgical success rate was $80 \%$.(Li, Lee et al. 2016) The Coblation lingual tonsil

249 removal technique proved to be an effective procedure in a cohort of Korean OSAS

250 patients with retroglossal obstruction. The mean $\mathrm{AHI}$ decreased significantly from

$25137.7 \pm 18.6$ to $18.7 \pm 14.8$ events/hour $(p<.001)$ and the success rate was $55.6 \%$. (Wee,

252 Tan et al. 2015) Another study compared combined coblation endoscopic lingual 
253 lightening and relocation pharyngoplasty to relocation pharyngoplasty alone in OSAS

254 patients (AHI>20, Friedman stage III), and reported that combined surgery had better

255 improvement in $\mathrm{AHI}(-65.5$ vs $-53.2 ; \mathrm{p}=.047)$ and higher surgical success rate than

256 relocation pharyngoplasty alone (73\% vs 50\%; p=.04).(Li, Lee et al. 2016)

257

258 O'Malley et al. developed a minimally invasive surgical procedure for management of

259 tongue base neoplasms by using robotic surgical instruments.(O'Malley, Weinstein et al.

260 2006) Trans-oral robotic surgery (TORS) can offer clear 3D visualization and gain

261 adequate access to tongue base, larynx and hypopharynx and provide meticulous tissue

262 resection. A preliminary study in 2010 conducted by Vicini et al. reported that TORS for

263 tongue base resection in OSAS patients is practical and well tolerated. Ten patients were

264 included and the AHI (mean \pm SD) decreased from $38.3 \pm 23.5$ to $20.6 \pm 17.3$

265 events/hour.(Vicini, Dallan et al. 2010) Further study for demonstration of the feasibility

266 of TORS performed in forty four patients with OSAS reported significant improvement of

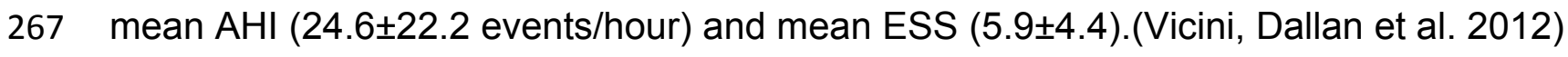

268 The latest systematic review and meta-analysis by Meccariello et al. concluded that

269 TORS seems to be a promising and safe technology for the management of OSAS and

270 the mean failure rate was $34.4 \%$ (29.5-46.2\%).(Meccariello, Cammaroto et al. 2017) 
271

272 A study by Friedman et al. in 2012 was thought to be the first comparative study for

273 comparison of coblation and TORS in OSAS treatment.(Friedman, Hamilton et al. 2012)

274 It compared the effectiveness of TORS with that of coblation assisted submucosal

275 minimally invasive lingual excision (SMILE). All the patients in the study received

276 concomitant z-palatoplasty. The AHI (mean \pm SD) reduction in the TORS and SMILE

277 groups were $60.5 \% \pm 24.9 \%$ and $32.0 \% \pm 43.3 \%(p=.012)$, respectively. The success

278 rate in the TORS and SMILE groups were $66.7 \%$ and $45.5 \%$, respectively; the between-

279 group difference was not significant $(p=.135)$. However, the techniques used by each

280 group were different and not completely comparable.

281

282 To the best of our knowledge, there is few finely matched studies regarding the

283 treatment efficacy and safety between TORS and coblation adopting similar technique in

284 tongue base resection. Our retrospective comparison of TORS with coblation in the

285 treatment of OSAS patients with multilevel obstruction found that both groups had similar

286 surgical results.

287

288 The demographics and preoperative polysomnographic data did not differ significantly 
289 between the two groups (Table 2) at baseline. According to the within-group outcomes

290 showed in Table 3, statistically significant improvement of apnea-hyponea index (AHI),

291 apnea index (Al), Epworth Sleepiness scale (ESS) and minimum oxygen saturation (min-

292 SpO2) were noted in both the TORS and coblation groups. It confirmed that either TORS

293 tongue base resection or coblation assisted tongue base resection combined with

294 concomitant uvuolopalatoplsty can offer reliable surgical results. The cumulative time

295 percentage with SpO2 < 90\% (CT90) were decreased in both groups but only

296 significantly reduced in the TORS group which could be related to small sample size or

297 poor correlation of CT90 to AHI.(Chung, Liao et al. 2012)

298

299 As detailed in Table 4, the mean reduction of AHI, Al, ESS, CT90 and mean

300 improvement of min-SpO2 were similar for the TORS and coblation groups. The rate of

301 surgical success in the TORS group were comparable to the coblation group ( $50 \%$ vs

$30258 \%, p=.611)$. Hwang et al. compared the tongue base coblation resection to TORS in

303 OSAS patients and both groups were in combination with lateral pharyngoplasty.(Hwang,

304 Kim et al. 2017) They reported that the surgical success rates did not differ significantly

305 between the two groups $(56.3 \%$ in TORS vs $62.1 \%$ in coblation, $p=.711)$. Our success

306 rates are lower than those in that study. However, the preoperative BMI of patients in 
307 that study was lower than in our study group (TORS group $=28.2 \pm 3.8 \mathrm{~kg} / \mathrm{m}^{2}$; coblation

308 group $\left.=27.4 \pm 5.6 \mathrm{~kg} / \mathrm{m}^{2}, \mathrm{p}=.645\right)$.. Moreover, preoperative mean ESS were lower

309 (TORS group $=11.1 \pm 4.7$; coblation group $=10.9 \pm 5.2, p=.917)$ and mean min-SpO2

310 were higher $($ TORS group $=73.8 \pm 6.8 \%$; coblation group $=74.0 \pm 10.0 \%, p=.951)$ in

311 their study than those in our study group, which might suggest the severity of OSAS

312 is greater in our patients. In our study, the mean pain scores (numeric rating scales) on

313 the first postoperative day were comparable in both groups (TORS group $=2.8 \pm 0.9$;

314 coblation group $=2.5 \pm 0.7 ; p=.533$ ). In the TORS group, the length of stay in hospital

315 was longer compared with the coblation group $(p=.002)$. There was no major

316 complication in either group. The rates of minor complication were higher in the TORS

317 group (50\%) than that of the coblation group (35.3\%) without statistical significance.

318 According to a review article, slightly better outcomes were observed in TORS compared

319 to coblation, but the higher rate of minor complications and the significant costs of TORS

320 are two aspects which surgeons will need to consider. (Cammaroto, Montevecchi et al.

$3212017)$

322

323 This study has some limitations. First, the retrospective analysis used in this study is a

324 possible source for selection bias by patients' preferences even after shared decision 
325 makings and routine surgical treatment strategy explanation. Second, it is difficult to

326 make comparisons among studies because of different surgical techniques utilized by

327 TORS (e.g. midline posterior glossectomy(Lin, Rowley et al. 2015), lingual

328 tonsillectomy(Muderris, Sevil et al. 2015)) and coblation (e.g. midline posterior

329 glossectomy(Li, Lee et al. 2016), SMILE(Friedman, Soans et al. 2008), channelling of

330 the tongue(Zhang and Liu 2014), Interstitial injections with needle coblation(Hou, Hu et

331 al. 2012)). Third, non-parametric approach is that we are unable to adjust for potential

332 confounders. In the future, prospective, randomized, controlled trials that incorporate

333 similar surgical technique will be needed to evaluate the efficacy of TORS compared with

334 coblation tongue base reduction. Moreover, studies providing long-term results in the

335 treatment of OSAS are also warranted.

336

337 Conclusion

338 TORS resulted in comparable objective and subjective outcomes compared to coblation

339 assisted tongue base reduction surgery in OSAS patients. Multilevel surgery with either

340 TORS or coblation tongue base reduction combined with uvulopalatoplasty is effective in

341 reducing disease severity in moderate-to-severe OSAS cases. 
344 The authors would like to thank all colleagues of department of Otolaryngology Head and

345 Neck Surgery in China Medical University Hospital who provided insight and expertise

346 that greatly assisted the research. This work was also supported by China Medical

347 University (No. CMU-102-bc3 and DMR-107-040).

\section{References}

350 Cammaroto, G., F. Montevecchi, G. D'Agostino, E. Zeccardo, C. Bellini, B. Galletti, M. Shams, H.

351 Negm and C. Vicini (2017). "Tongue reduction for OSAHS: TORSs vs coblations, technologies vs 352 techniques, apples vs oranges." Eur Arch Otorhinolaryngol 274(2): 637-645.

353 Choi, J. H., S. H. Cho, S. N. Kim, J. D. Suh and J. H. Cho (2016). "Predicting Outcomes after

354 Uvulopalatopharyngoplasty for Adult Obstructive Sleep Apnea: A Meta-analysis." Otolaryngol 355 Head Neck Surg 155(6): 904-913.

356 Chung, F., P. Liao, H. Elsaid, S. Islam, C. M. Shapiro and Y. Sun (2012). "Oxygen desaturation

357 index from nocturnal oximetry: a sensitive and specific tool to detect sleep-disordered

358 breathing in surgical patients." Anesth Analg 114(5): 993-1000.

359 Friedman, M., C. Hamilton, C. G. Samuelson, K. Kelley, D. Taylor, K. Pearson-Chauhan, A. Maley, 360 R. Taylor and T. K. Venkatesan (2012). "Transoral robotic glossectomy for the treatment of 361 obstructive sleep apnea-hypopnea syndrome." Otolaryngol Head Neck Surg 146(5): 854-862.

362 Friedman, M., A. M. Salapatas and L. B. Bonzelaar (2017). "Updated Friedman Staging System 363 for Obstructive Sleep Apnea." Adv Otorhinolaryngol 80: 41-48.

364 Friedman, M., R. Soans, B. Gurpinar, H. C. Lin and N. Joseph (2008). "Evaluation of submucosal 365 minimally invasive lingual excision technique for treatment of obstructive sleep

366 apnea/hypopnea syndrome." Otolaryngol Head Neck Surg 139(3): 378-384; discussion 385.

367 Friedman, M., H. Tanyeri, M. La Rosa, R. Landsberg, K. Vaidyanathan, S. Pieri and D. Caldarelli 368 (1999). "Clinical predictors of obstructive sleep apnea." Laryngoscope 109(12): 1901-1907. 369 Hou, T., S. Hu and X. Jiang (2012). "Tongue coblation via the ventral approach for obstructive 370 sleep apnea-hypopnea syndrome surgery." Laryngoscope 122(11): 2582-2586.

371 Hwang, C. S., J. W. Kim, J. W. Kim, E. J. Lee, C. H. Kim, J. H. Yoon and H. J. Cho (2017). 
372 "Comparison of robotic and coblation tongue base resection for obstructive sleep apnoea." Clin 373 Otolaryngol.

374 Kezirian, E. J., W. Hohenhorst and N. de Vries (2011). "Drug-induced sleep endoscopy: the VOTE 375 classification." Eur Arch Otorhinolaryngol 268(8): 1233-1236.

376 Khan, A., K. Ramar, S. Maddirala, O. Friedman, J. F. Pallanch and E. J. Olson (2009).

377 "Uvulopalatopharyngoplasty in the management of obstructive sleep apnea: the mayo clinic 378 experience." Mayo Clin Proc 84(9): 795-800.

379 Kotecha, B. T. and A. C. Hall (2014). "Role of surgery in adult obstructive sleep apnoea." Sleep 380 Med Rev 18(5): 405-413.

381 Koutsourelakis, I., F. Safiruddin, M. Ravesloot, S. Zakynthinos and N. de Vries (2012). "Surgery

382 for obstructive sleep apnea: sleep endoscopy determinants of outcome." Laryngoscope 122(11): $383 \quad 2587-2591$.

384 Li, H. Y., L. A. Lee and E. J. Kezirian (2016). "Coblation endoscopic lingual lightening (CELL) for 385 obstructive sleep apnea." Eur Arch Otorhinolaryngol 273(1): 231-236.

386 Li, H. Y., L. A. Lee and E. J. Kezirian (2016). "Efficacy of Coblation Endoscopic Lingual Lightening 387 in Multilevel Surgery for Obstructive Sleep Apnea." JAMA Otolaryngol Head Neck Surg 142(5): 388 438-443.

389 Lin, H.-C., M. Friedman, H.-W. Chang, L. Bonzelaar, A. M. Salapatas, M.-C. Lin and K.-T. Huang 390 (2017). "Minimally Invasive, Single-Stage, Multilevel Surgery for Obstructive Sleep Apnea in 391 Asian PatientsMinimally Invasive Surgery for Obstructive Sleep Apnea in Asian 392 PatientsMinimally Invasive Surgery for Obstructive Sleep Apnea in Asian Patients." JAMA 393 Otolaryngology-Head \& Neck Surgery 143(2): 147-154.

394 Lin, H. S., J. A. Rowley, A. J. Folbe, G. H. Yoo, M. S. Badr and W. Chen (2015). "Transoral robotic 395 surgery for treatment of obstructive sleep apnea: factors predicting surgical response."

396 Laryngoscope 125(4): 1013-1020.

397 Lucia Spicuzza, D. C. a. G. D. M. (2015). "Obstructive sleep apnoea syndrome and its 398 management." Ther Adv Chronic Dis 6(5): 273-285.

399 Meccariello, G., G. Cammaroto, F. Montevecchi, P. T. Hoff, M. E. Spector, H. Negm, M. Shams, C. 400 Bellini, E. Zeccardo and C. Vicini (2017). "Transoral robotic surgery for the management of 401 obstructive sleep apnea: a systematic review and meta-analysis." Eur Arch Otorhinolaryngol 402 274(2): 647-653.

403 Muderris, T., E. Sevil, S. Bercin, F. Gul and M. Kiris (2015). "Transoral robotic lingual 404 tonsillectomy in adults: preliminary results." Acta Otolaryngol 135(1): 64-69.

405 O'Malley, B. W., Jr., G. S. Weinstein, W. Snyder and N. G. Hockstein (2006). "Transoral robotic 406 surgery (TORS) for base of tongue neoplasms." Laryngoscope 116(8): 1465-1472.

407 Punjabi, N. M. (2008). "The epidemiology of adult obstructive sleep apnea." Proc Am Thorac Soc 
408 5(2): 136-143.

409 Semelka, M., J. Wilson and R. Floyd (2016). "Diagnosis and Treatment of Obstructive Sleep

410 Apnea in Adults." Am Fam Physician 94(5): 355-360.

411 Terris, D. J., M. M. Hanasono and Y. C. Liu (2000). "Reliability of the Muller maneuver and its

412 association with sleep-disordered breathing." Laryngoscope 110(11): 1819-1823.

413 Thaler, E. R., C. H. Rassekh, J. M. Lee, G. S. Weinstein and B. W. O'Malley, Jr. (2016). "Outcomes

414 for multilevel surgery for sleep apnea: Obstructive sleep apnea, transoral robotic surgery, and

415 uvulopalatopharyngoplasty." Laryngoscope 126(1): 266-269.

416 Toh, S. T., H. J. Han, H. N. Tay and K. L. Kiong (2014). "Transoral robotic surgery for obstructive

417 sleep apnea in Asian patients: a Singapore sleep centre experience." JAMA Otolaryngol Head

418 Neck Surg 140(7): 624-629.

419 Vicini, C., I. Dallan, P. Canzi, S. Frassineti, M. G. La Pietra and F. Montevecchi (2010). "Transoral

420 robotic tongue base resection in obstructive sleep apnoea-hypopnoea syndrome: a preliminary

421 report." ORL J Otorhinolaryngol Relat Spec 72(1): 22-27.

422 Vicini, C., I. Dallan, P. Canzi, S. Frassineti, A. Nacci, V. Seccia, E. Panicucci, M. Grazia La Pietra, F.

423 Montevecchi and M. Tschabitscher (2012). "Transoral robotic surgery of the tongue base in

424 obstructive sleep Apnea-Hypopnea syndrome: anatomic considerations and clinical

425 experience." Head Neck 34(1): 15-22.

426 Vroegop, A. V., O. M. Vanderveken, A. N. Boudewyns, J. Scholman, V. Saldien, K. Wouters, M. J.

427 Braem, P. H. Van de Heyning and E. Hamans (2014). "Drug-induced sleep endoscopy in sleep-

428 disordered breathing: report on 1,249 cases." Laryngoscope 124(3): 797-802.

429 Wee, J. H., K. Tan, W. H. Lee, C. S. Rhee and J. W. Kim (2015). "Evaluation of coblation lingual

430 tonsil removal technique for obstructive sleep apnea in Asians: preliminary results of surgical

431 morbidity and prognosticators." Eur Arch Otorhinolaryngol 272(9): 2327-2333.

432 Zhang, Q. and D. Liu (2014). "Coblation-channelling for the tongue." Lin Chung Er Bi Yan Hou

433 Tou Jing Wai Ke Za Zhi 28(6): 386-388.

434

Peer) reviewing PDF | (2019:03:36243:2:1:NEW 21 Aug 2019) 


\section{Table $\mathbf{1}$ (on next page)}

Inclusion criteria

$\mathrm{AHI}=$ Apnea-Hypopnea Index; DISE = Drug-Induced Sleep Endoscopy; CPAP = Continuous positive airway pressure 
Table 1:

Inclusion criteria

$\geq 18$ years old

Symptoms of obstructive sleep apnea syndrome (snoring, disrupted sleep, daytime sleepiness)

Preoperative $\mathrm{AHI}>10$

Friedman tongue position grade 3 or 4

Partial or complete retropalatal and retroglossal collapse in Muller's maneuver and DISE

Cannot tolerate CPAP

$\mathrm{AHI}=$ Apnea-Hypopnea Index; DISE = Drug-Induced Sleep Endoscopy; CPAP = Continuous positive airway pressure 1 


\section{Table 2 (on next page)}

Demographics, Baseline data of the 2 groupst

$\mathrm{BMI}=$ body mass index (weight in kilograms devided by height in meters squared); FTP = Friedman tongue position; DISE = Drug-Induced Sleep Endoscopy; ESS = Epworth Sleepiness scale; $\mathrm{AHI}=$ Apnea-Hypopnea index; $\mathrm{Al}=$ Apnea index; Min-SpO2 = minimum oxygen saturation; CT90 = cumulative time percentage with $\mathrm{SpO} 2<90 \%$ † All values are presented as mean \pm standard deviation 
TABLE 2.

Demographics, Baseline data of the 2 groups $\dagger$

\begin{tabular}{lccc}
\hline \hline & $\begin{array}{c}\text { TORS group } \\
(\mathrm{n}=16)\end{array}$ & $\begin{array}{c}\text { Coblation group } \\
(\mathrm{n}=17)\end{array}$ & p value \\
\hline age, years & $39.4 \pm 12.3$ & $38.7 \pm 11.5$ & .861 \\
Male, $\mathrm{n}(\%)$ & $15(93.8)$ & $13(76.5)$ & .335 \\
BMI, kg/m ${ }^{2}$ & $28.2 \pm 3.8$ & $27.4 \pm 5.6$ & .645 \\
Tonsil grade & $2.0 \pm 1.3$ & $1.9 \pm 0.8$ & .748 \\
FTP & $3.4 \pm 0.6$ & $3.4 \pm 0.6$ & .918 \\
Friedman stage & $2.5 \pm 0.6$ & $2.7 \pm 0.6$ & .340 \\
Grade of collapse in Muller maneuver & & & \\
\multicolumn{1}{c}{ Retropalatal area } & $3.2 \pm 0.9$ & $3.6 \pm 0.6$ & .147 \\
$\quad$ Retroglossal area & $2.6 \pm 0.9$ & $2.3 \pm 0.8$ & .260 \\
Grade of collapse in DISE $\quad$ Velum & & & .428 \\
$\quad$ Oropharynx & $1.9 \pm 0.3$ & $1.8 \pm 0.4$ & .624 \\
$\quad$ Tongue base & $1.5 \pm 0.5$ & $1.6 \pm 0.5$ & .611 \\
$\quad$ Epiglottis & $1.4 \pm 0.5$ & $1.5 \pm 0.5$ & .460 \\
ESS & $0.5 \pm 0.6$ & $0.4 \pm 0.5$ & .917 \\
AHI, events/hour & $11.1 \pm 4.7$ & $10.9 \pm 5.2$ & .517 \\
Al, events/hour & $50.5 \pm 19.6$ & $44.9 \pm 28.8$ & .816 \\
Min-SpO2, \% & $33.3 \pm 19.2$ & $31.3 \pm 26.8$ & .951 \\
CT90, \% & $73.8 \pm 6.8$ & $74.0 \pm 10.0$ & .705 \\
\hline \hline
\end{tabular}

$\mathrm{BMI}=$ body mass index (weight in kilograms devided by height in meters squared); FTP = Friedman tongue position; DISE = Drug-Induced Sleep Endoscopy; ESS = Epworth Sleepiness scale; $\mathrm{AHI}=$ Apnea-Hypopnea index; $\mathrm{Al}=$ Apnea index; $\mathrm{Min}-\mathrm{SpO} 2=$ minimum oxygen saturation; $\mathrm{CT} 90=$ cumulative time percentage with $\mathrm{SpO} 2<90 \%$ $\dagger$ All values are presented as mean \pm standard deviation 


\section{Table 3(on next page)}

Within-group comparison of the treatment outcomest

$\mathrm{AHI}=$ Apnea-Hypopnea index; $\mathrm{Al}=$ Apnea index $; \mathrm{ESS}=$ Epworth Sleepiness scale; Min-SpO2 = minimum oxygen saturation; CT90 = cumulative time percentage with $\mathrm{SpO} 2<90 \%$

$\dagger$ All values are presented as mean \pm standard deviation $* p<.05$ is considered statistically significant 
TABLE 3.

Within-group comparison of the treatment outcomes $†$

\begin{tabular}{|c|c|c|c|c|c|c|}
\hline & \multicolumn{3}{|c|}{$\begin{array}{c}\text { TORS group } \\
(\mathrm{n}=16)\end{array}$} & \multicolumn{3}{|c|}{$\begin{array}{l}\text { Coblation group } \\
\qquad(\mathrm{n}=17)\end{array}$} \\
\hline & Preoperative & Postoperative & $p$ value & Preoperative & Postoperative & $\mathrm{p}$ value \\
\hline $\mathrm{AHI}$ & $50.5 \pm 19.6$ & $25.5 \pm 19.5$ & $.002^{*}$ & $44.8 \pm 28.8$ & $25.5 \pm 23.3$ & $.005^{*}$ \\
\hline $\mathrm{Al}$ & $33.3 \pm 19.2$ & $16.5 \pm 17.5$ & $.014^{*}$ & $31.4 \pm 26.9$ & $12.7 \pm 22.0$ & $.004^{*}$ \\
\hline ESS & $11.1 \pm 4.7$ & $7.6 \pm 3.6$ & $<.001^{*}$ & $10.9 \pm 5.2$ & $8.1 \pm 5.5$ & $.017^{*}$ \\
\hline Min-SpO2 & $73.8 \pm 6.8$ & $83.8 \pm 5.6$ & $<.001^{*}$ & $74.0 \pm 10.0$ & $80.7 \pm 12.6$ & $.045^{*}$ \\
\hline СT90 & $15.1 \pm 14.5$ & $5.7 \pm 7.6$ & $.005^{*}$ & $13.2 \pm 15.2$ & $8.2 \pm 18.3$ & .183 \\
\hline
\end{tabular}

$\mathrm{AHI}=$ Apnea-Hypopnea index; $\mathrm{Al}=$ Apnea index; $\mathrm{ESS}=$ Epworth Sleepiness scale; Min-SpO2 = minimum oxygen saturation; $\mathrm{CT} 90=$ cumulative time percentage with $\mathrm{SpO} 2<90 \%$

$\dagger$ All values are presented as mean \pm standard deviation

${ }^{*} p<.05$ is considered statistically significant 


\section{Table 4(on next page)}

Between-groups comparison of the treatment outcomes

$\mathrm{SD}=$ standard deviation; $\mathrm{AHI}=$ Apnea-Hypopnea index; $\mathrm{Al}=$ Apnea index; ESS = Epworth

Sleepiness scale; Min-SpO2 = minimum oxygen saturation; CT90 = cumulative time percentage with SpO2 < 90\%; NRS = numerical rating scale 
TABLE 4.

Between-groups comparison of the treatment outcomes

\begin{tabular}{lccc}
\hline \hline & $\begin{array}{c}\text { TORS group } \\
(\mathrm{n}=16)\end{array}$ & $\begin{array}{c}\text { Coblation group } \\
(\mathrm{n}=17)\end{array}$ & $\mathrm{p}$ value \\
\hline AHI reduction (events/h) & $24.9 \pm 26.5$ & $19.4 \pm 24.8$ & .631 \\
Al reduction(events/h) & $16.7 \pm 23.9$ & $18.7 \pm 22.7$ & .657 \\
ESS reduction & $3.8 \pm 6.6$ & $3.1 \pm 9.2$ & .873 \\
Min-SpO2 improvement & $10.0 \pm 7.7$ & $6.7 \pm 12.6$ & .363 \\
CT90 reduction & $9.3 \pm 11.4$ & $4.9 \pm 14.7$ & .510 \\
Success rate, $\mathrm{n}(\%)$ & $8(50.0)$ & $10(58.8)$ & .611 \\
Day 1 pain score(NRS) & $2.8 \pm 0.9$ & $2.5 \pm 0.7$ & .533 \\
Hospital stay (days) & $5.5 \pm 1.2$ & $4.4 \pm 0.7$ & .004 \\
Major complication, $\mathrm{n}(\%)$ & $0(0)$ & $0(0)$ & \\
Minor complication, $\mathrm{n}(\%)$ & $8(50.0)$ & $6(35.3)$ & .393 \\
\hline \hline
\end{tabular}

$\mathrm{AHI}=$ Apnea-Hypopnea index; $\mathrm{Al}=$ Apnea index; ESS $=$ Epworth Sleepiness scale; Min-SpO2 = minimum oxygen saturation; $\mathrm{CT} 90=$ cumulative time percentage with $\mathrm{SpO} 2<90 \%$; NRS $=$ numerical rating scale 
Figure 1

The study flow diagram

\section{Enrollment}

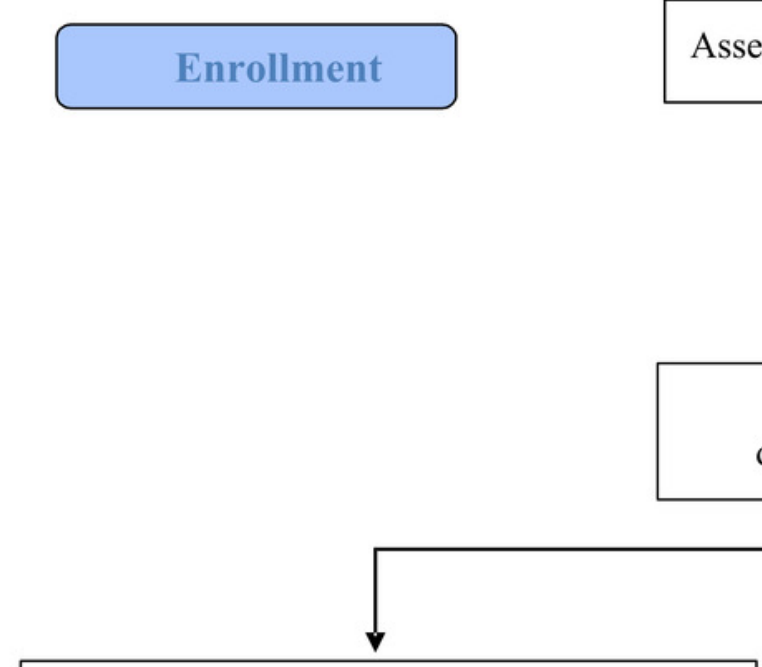

Allocated to intervention $(\mathrm{n}=18)$

Received allocated intervention $(\mathrm{n}=18)$
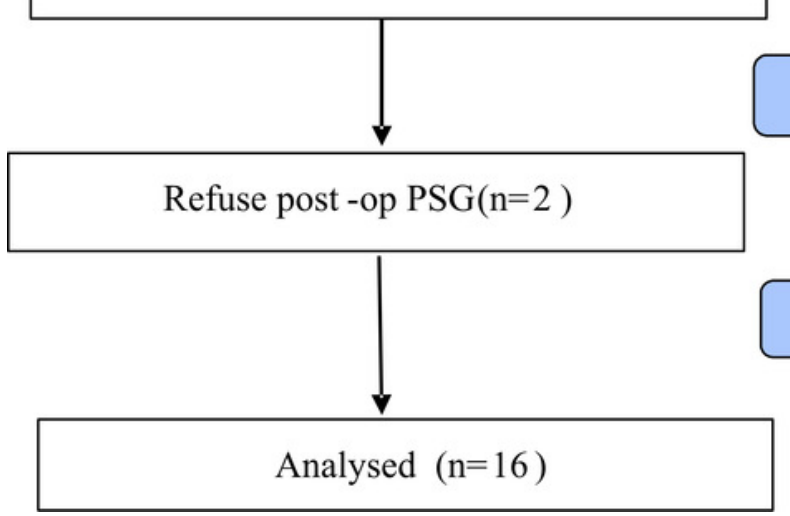

Excluded $(n=4)$

Not meeting inclusion criteria $(\mathrm{n}=2)$

Other reasons (chose other surgical methods; $\mathrm{n}=2$ )
Allocated by Shared decision making $(\mathrm{n}=36)$

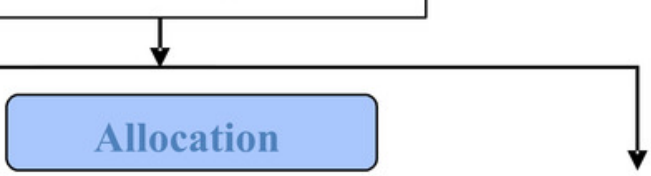

Allocated to intervention $(\mathrm{n}=18)$ Received allocated intervention $(n=18)$
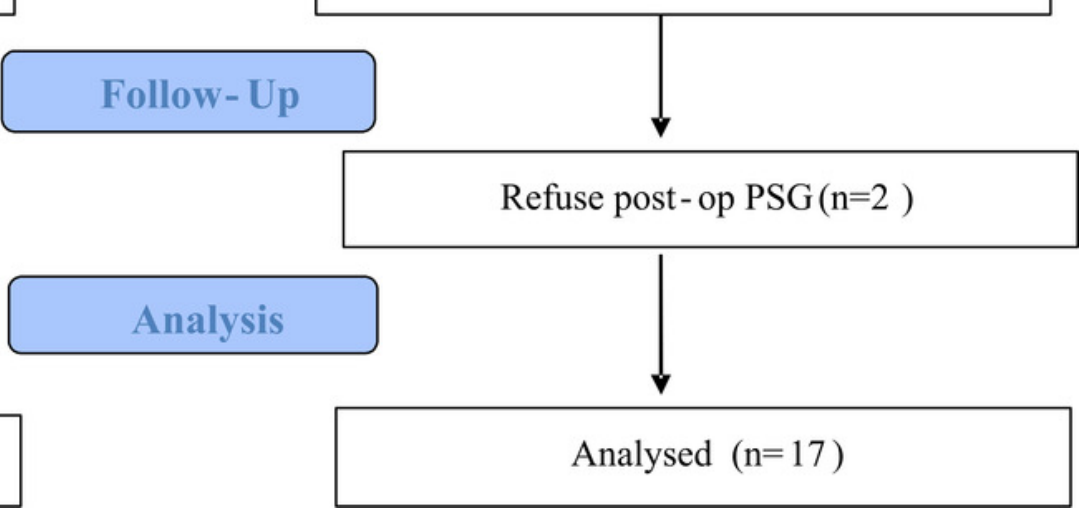
Figure 2

The treatment outcome of ESS between two groups

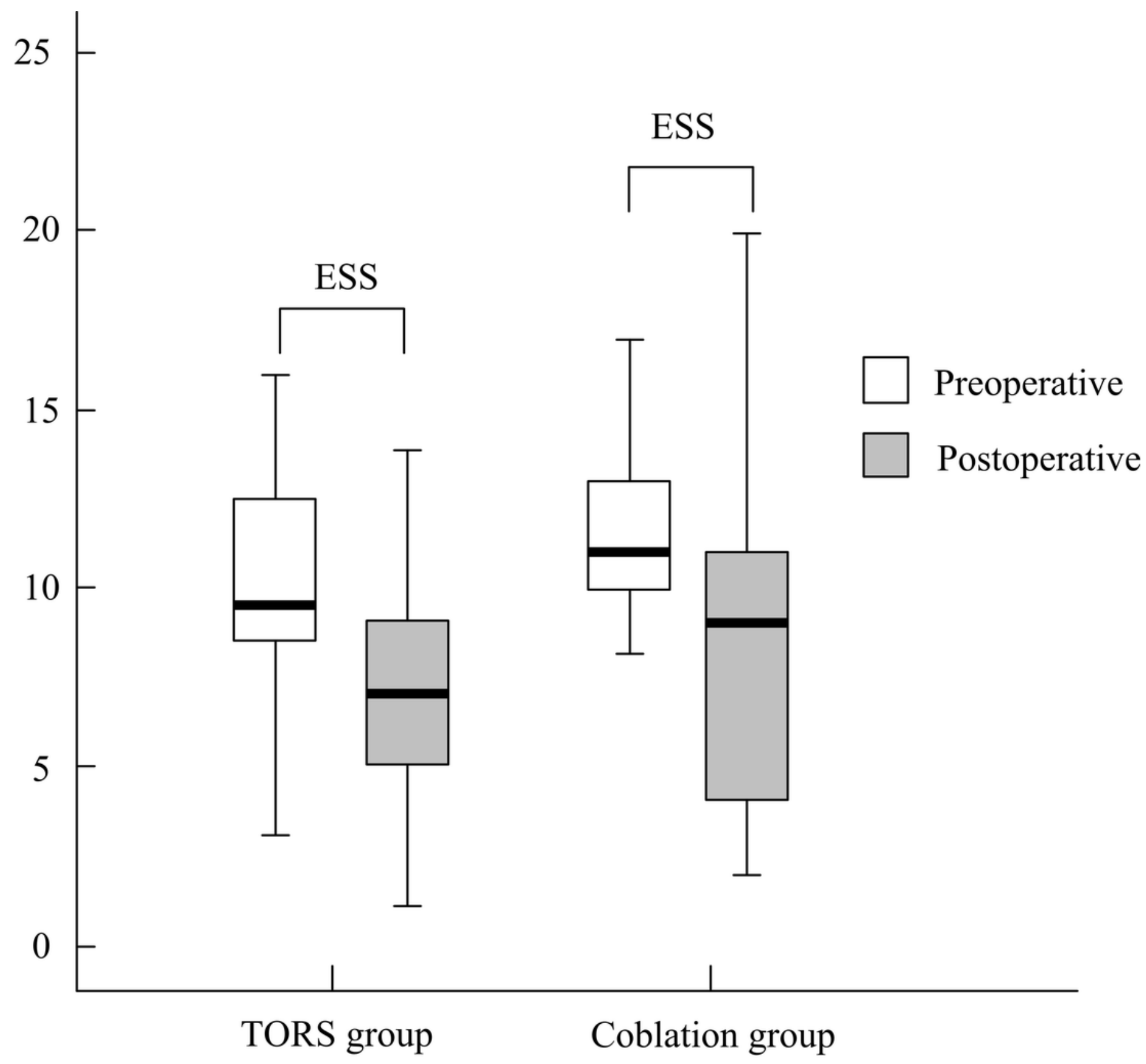


Figure 3

Individual AHI decrease in TORS (A) and Coblation (B) groups
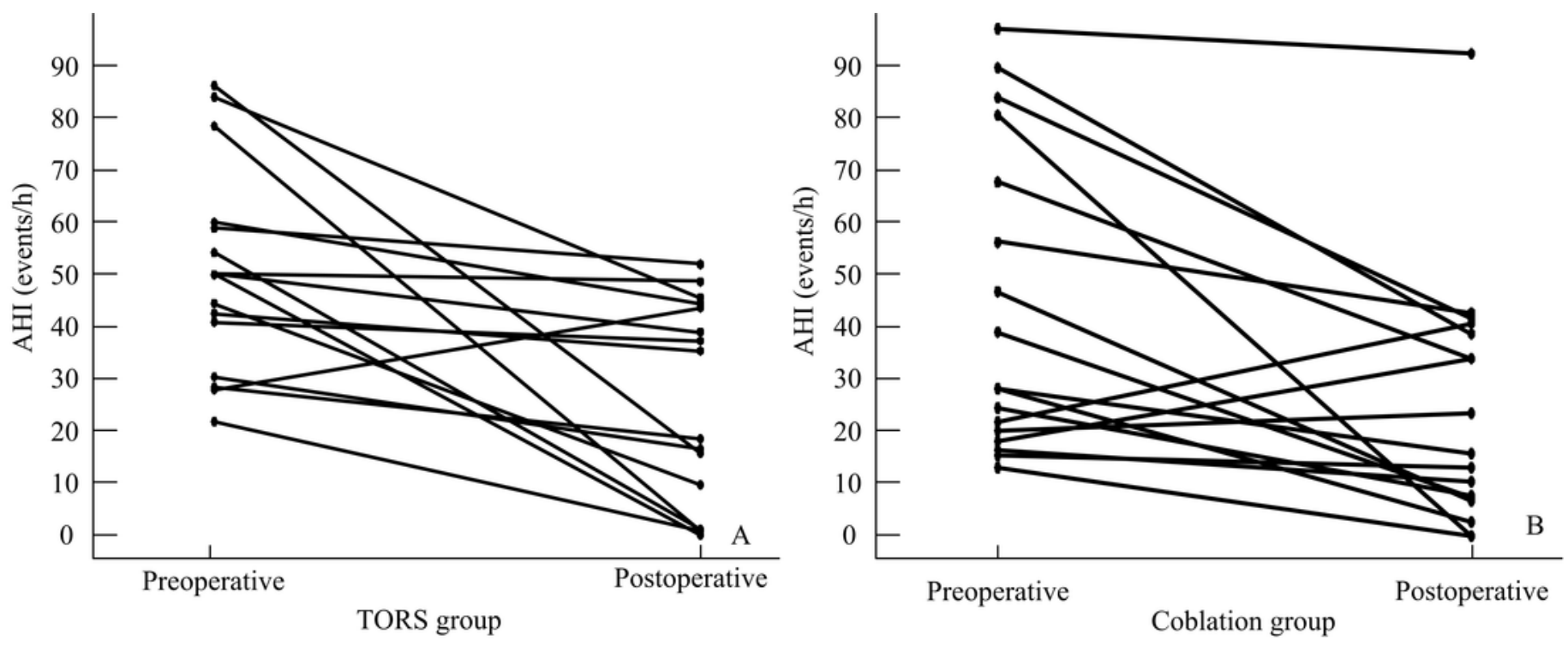
Figure 4

The treatment outcome of $\mathrm{AHI}$ and $\mathrm{Al}$ between two groups

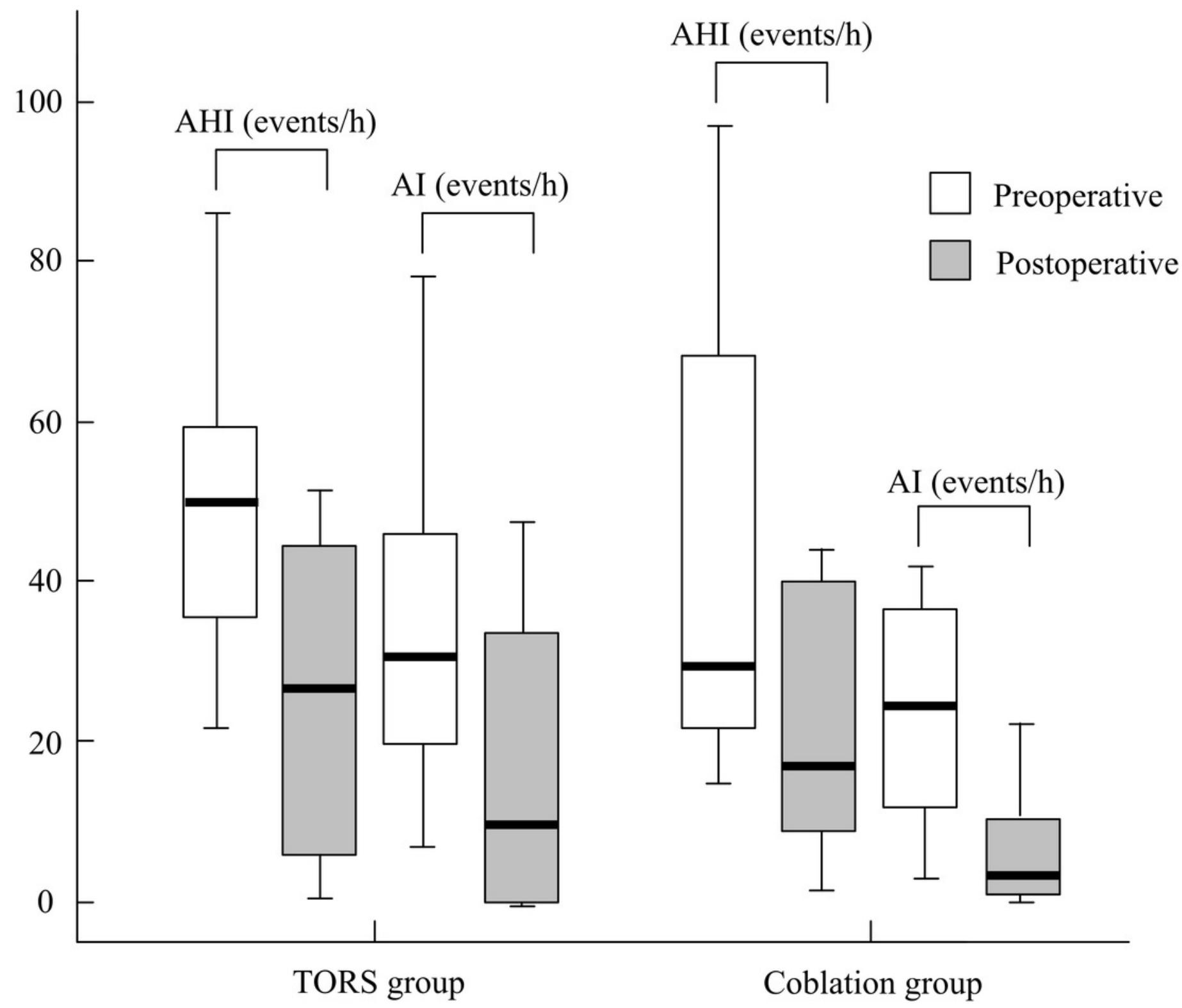


Figure 5

The treatment outcome of min-SpO2 and CT 90 between two groups

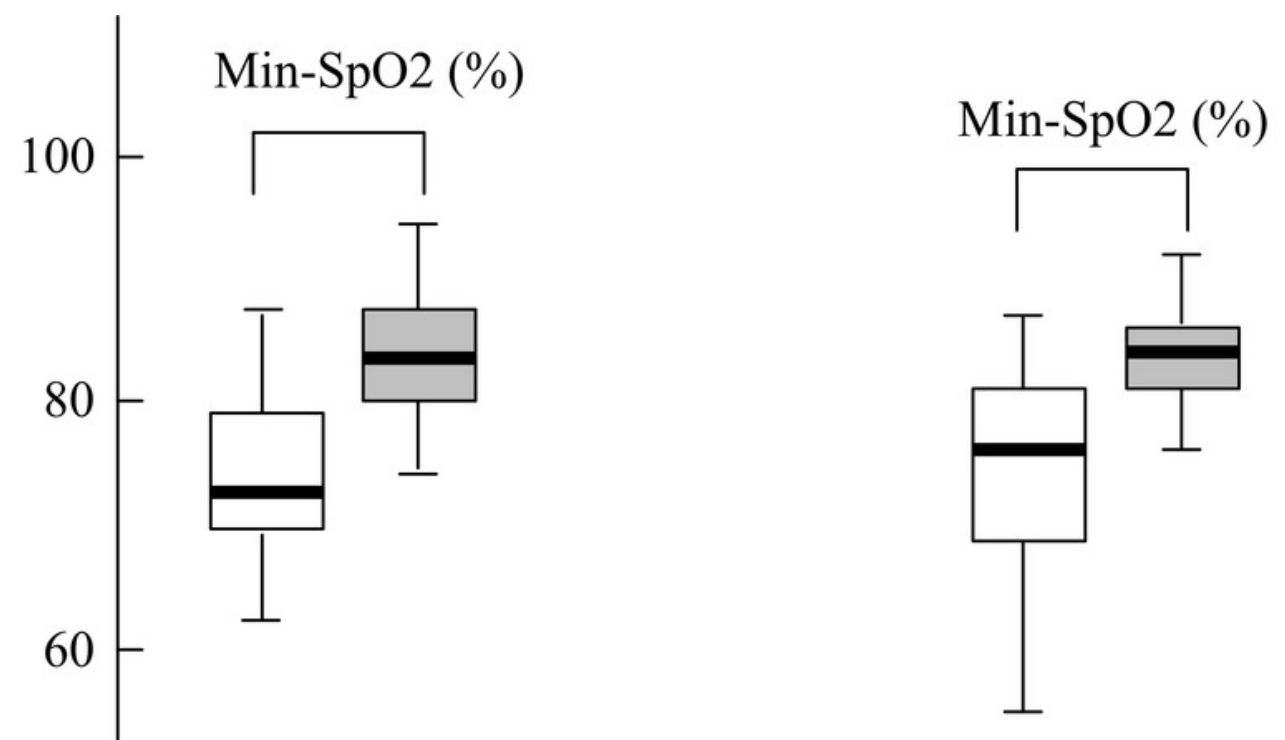

CT90(\%)

$40-$
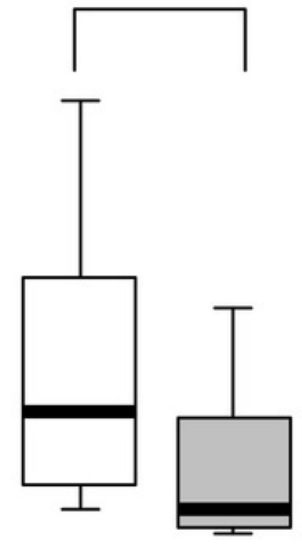

TORS group

\section{1}

Coblation group $\square$ Preoperative

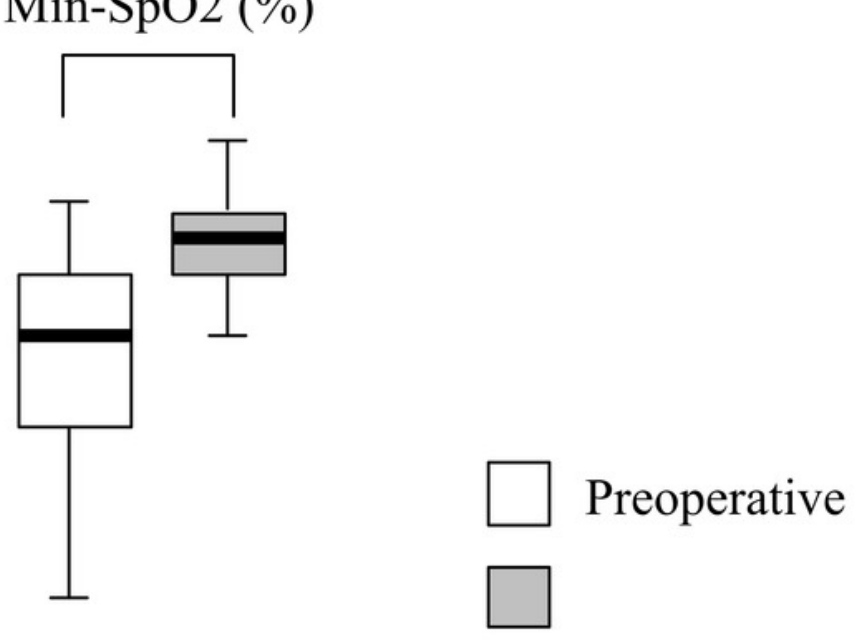

CT90(\%)
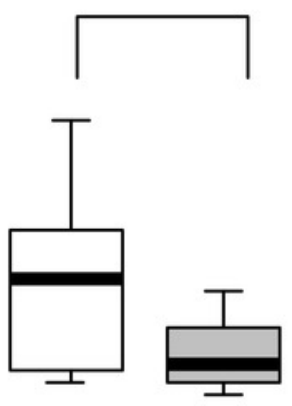